\title{
Assessing COVID-19 cohorting strategies in a UK district general hospital during the first wave of COVID-19
}

Authors: Tim Davies, ${ }^{\mathrm{A}}$ Tamsin Cargill, ${ }^{\mathrm{A}}$ Robert Shaw, ${ }^{\mathrm{A}}$ Tony Ellis, ${ }^{\mathrm{A}}$ Katie Jeffery ${ }^{\mathrm{A}}$ and Tri Wangrangsimakul ${ }^{\mathrm{A}}$

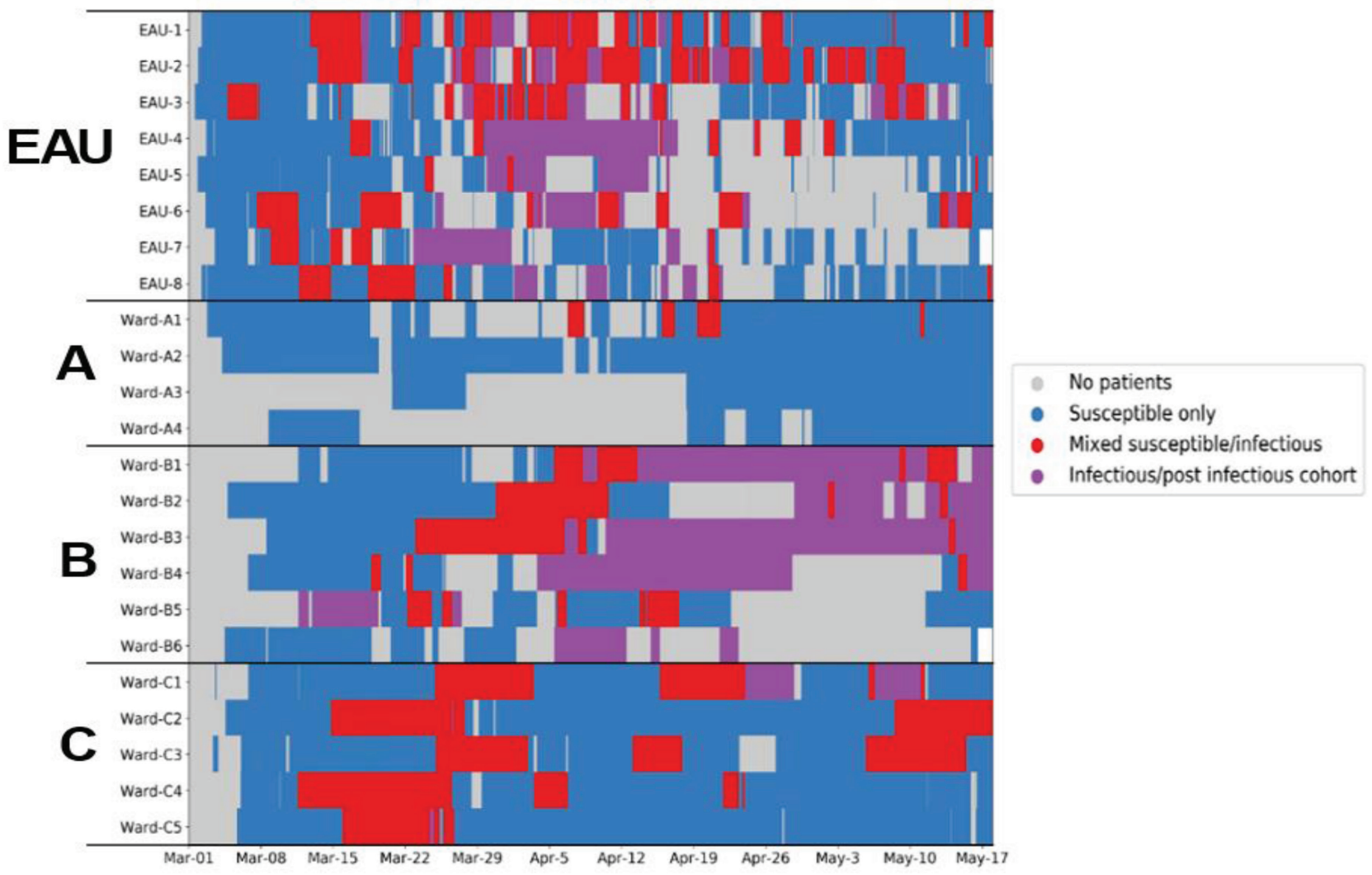

Fig 1. Bay occupancy/sharing across Horton General Hospital, 01 March 2020 to 15 May 2020.

\section{Introduction}

Patient-to-patient transmission has a well-recognised role in nosocomial COVID-19 infections, ${ }^{1}$ but identifying and isolating infectious individuals is challenging, due to limitations in the speed and accuracy of testing. ${ }^{2}$ To mediate

Author: ${ }^{A}$ Oxford University Hospitals NHS Foundation Trust, Oxford, UK this, many hospitals attempted to cohort patients based on suspicion of COVID-19 infection. ${ }^{3}$ We aimed to assess whether this strategy was successful at a district general hospital (DGH) in Oxfordshire.

\section{Methods}

We retrospectively analysed records of all medical patients admitted to the Horton General Hospital (HGH), Banbury between 1 March and 18 May 2020. HGH is a small DGH that is part of the Oxford University Hospitals NHS Foundation Trust. At HGH, all medical patients are seen in either the 
Table 1. Exposure in emergency assessment unit

$\begin{array}{llllll}\text { Bay } & \begin{array}{l}\text { Total } \\ \text { patient } \\ \text { hours }\end{array} & \begin{array}{l}\text { Infectious } \\ \text { patient } \\ \text { hours }\end{array} & \begin{array}{l}\text { Susceptible } \\ \text { patient } \\ \text { hours }\end{array} & \begin{array}{l}\text { Susceptible patient hours } \\ \text { exposed to infectious } \\ \text { patients }\end{array} & \begin{array}{l}\text { Hours during the study in which the bay } \\ \text { contained both infectious and susceptible } \\ \text { patients (\% of 1872 } \nabla \text { total study timeframe) }\end{array} \\ \text { EAU-1 } & 5,114 & 1,070 & 3,921 & 1,123 & 590(32) \\ \text { EAU-2 } & 5,061 & 1,226 & 3,680 & 1,083 & 628(34) \\ \text { EAU-3 } & 3,243 & 713 & 2,356 & 617 & 340(18) \\ \text { EAU-4 } & 4,168 & 1,202 & 2,778 & 235 & 123(7) \\ \text { EAU-5 } & 2,607 & 691 & 1,909 & 51 & 36(2) \\ \text { EAU-6 } & 2,760 & 833 & 1,854 & 671 & 390(21) \\ \text { EAU-7 } & 1,608 & 271 & 1,155 & 154 & 154(8) \\ \text { EAU-8 } & 3,161 & 496 & 2,625 & 449 & 231(12)\end{array}$

emergency department or one of four medical wards including an emergency assessment unit (EAU). Patients are triaged at admission according to their likelihood of COVID-19 infection, with high/medium risk patients being admitted to EAU and low risk patients being admitted ward A. Patients requiring longer stays are then moved to ward B (high risk) or C (medium risk), depending on risk.

Using patient movement data, we identified periods when 'infectious' and 'susceptible' patients spent time in open bays together. We did this using several possible definitions of 'infectious', varying both the evidence required to diagnose COVID-19 (eg PCR swab positive vs symptoms plus radiological signs alone), and the period each case was infectious. For our primary definition, a case of COVID-19 infection (as determined by PCR) was infectious for 3 days prior to 10 days after their first recoded symptom/test result. Patients were susceptible if they had no evidence of prior COVID-19 infection.

\section{Results}

894/1016 (88\%) admissions (corresponding to 791 patients) occurring during the study period had inpatient stays on open bays in medical wards. Over half $(667 / 1,016 ; 66 \%)$ of these episodes were first admitted to EAU (medium/high), with only 122/1,016 (12\%) being admitted to ward A (low risk). Using our primary definition, 142 admissions (139 patients) had an infectious period. Our cohorting strategies for low-risk patients appeared successful, with bay sharing between infectious and susceptible patients occurring for only 146/12,906 (1.1\%) susceptible patient hours observed on ward A during the study (Fig 1). By contrast, there were significant periods of exposure in EAU (Table 1), with over a quarter of the hours susceptible patients spent in six of the eight bays being exposed to at least one other infectious patient. Similar patterns were seen on other wards irrespective of the definition of 'infectiousness' used. Worryingly, using our primary definition we found five patients that had shared a bay with an infectious patient 3-7 days prior to onset of their infection.

\section{Conclusion}

COVID-19 infectious and susceptible patients frequently shared bays during the first wave of the pandemic, despite cohorting patients with similar clinical risk together. The results support the need for rapid point of care COVID-19 tests to improve identification of infectious patients and increase the effectiveness of cohorting. This could reduce the risk of nosocomial/healthcare associated acquisition of COVID-19 in acute hospital settings.

\section{Conflicts of interest}

None declared.

\section{References}

1 Meredith LW, Hamilton WL, Warne B et al Rapid implementation of SARS-CoV-2 sequencing to investigate cases of healthcare associated COVID-19: a prospective genomic surveillance study. Lancet Infect Dis 2020;20:1263-72.

2 Tang YW, Schmitz JE, Persing DH et al. Laboratory diagnosis of COVID-19: Current issues and challenges. J Clin Microbiol 2020;58:e00512-20.

3 Patterson B, Marks M, Martinez-Garcia G et al. A novel cohorting and isolation strategy for suspected COVID-19 cases during a pandemic. J Hosp Infect 2020;105:632-7. 